Academic City University College - Accra Ghana

Society for Multidisciplinary \& Advanced Research Techniques (SMART) Africa

Tony Blair Institute for Global Change

FAIR Forward - Artificial Intelligence for All - Deutsche Gesellschaft für Internationale Zusammenarbeit (GIZ) GmbH

Accra Bespoke Multidisciplinary Innovations Conference (ABMIC)

\title{
Traffic Management System for Prioritizing Emergency Over Time and Density
}

\author{
Abasiama I. Silas, Faith M. Jonathan \& Otuekong E. Ekong \\ Department of Computer Science, \\ Akwa Ibom State University \\ Ikot Akpaden, Akwa Ibom State, Nigeria \\ E-mails: AbasiamaSilas@aksu.edu.ng1,fmfido95@gmail.com², OtuekongEkong@aksu.edu.ng3 \\ Phone Nos: +2348034765201, +2347034699473, +2348135406037
}

\section{Member Proceedings Citation Format}

Abasiama I. Silas, Faith M. Jonathan \& Otuekong E. Ekong (2021): Traffic Management System for Prioritizing Emergency Over Time and Density. Proceedings of the Accra Bespoke

Multidisciplinary Innovations Conference. University of Ghana/Academic City University College, Accra, Ghana. December 2021. Pp 23-36 www.isteams.net/ghanabespoke2021. DOI https://doi.org/ 10.22624/AIMS/ABMIC2021-V2-P3 


\title{
Traffic Management System for Prioritizing Emergency Over Time and Density
}

\author{
Abasiama I. Silas, Faith M. Jonathan \& Otuekong E. Ekong
}

\begin{abstract}
An analysis of the current traffic management system in Uyo, an urban city in Nigeria showed a heavy dependence on the use of traffic wardens. Where traffic control lights operate, they are based on fixed time allocation which often results in traffic congestion on the more operational lane and hinders the rescue work of first responders. This study proposes a traffic management system (TMS-ETD) that prioritizes the passage of Emergency vehicles over the lane with the longest waiting Time, and lastly the lane with highest traffic Density. The Dynamic Systems Development Method (DSDM) guided this work. A testbed to demonstrate the practicability of this model was designed using HC-SR505 IR sensor to measure traffic density and send the data to PIC 16F770A microcontroller, programmed using MikroC PRO version 6.0.0, for processing. The model was simulated using Proteus 8 professional version 8.6 where results showed a smoother passage of emergency vehicles necessary to safeguard lives and properties.
\end{abstract}

Keywords: Traffic control, Queuing analysis, Actuators, Infrared Image sensors, Wireless sensor networks

\section{1. INTRODUCTION}

Traffic congestion is a problem faced in many cities around the world. It typically occurs whenever the traffic demand approaches the maximum capacity of a road (especially near a road intersection) such that the traffic stream slows down, in many cases stops altogether, for long periods of time (Viswanathan \& Santhanam, 2013). It can be attributed to the high volume of vehicles, inadequate infrastructure and irrational siting of infrastructures. Traffic congestion leads to increased fuel costs, noise and environmental pollution. It hinders the rescue work of emergency vehicles like ambulances, firefighters, and police necessary to safeguard lives and property. In a recent study on the effect of traffic congestion in the mega-city of Lagos State, Nigeria, it cost individuals who own vehicles an extra $\$ 133,978.68$ per annum while those who use public transport spent an extra $\$ 79,039.40$ per annum as they commute between home and work (Ovadje, 2021). The report estimated the total loss at 14.12 million hours per day or \#3.834 trillion per annum.

In the early days, traffic was managed using traffic wardens or volunteers standing at the middle of road intersections. Being humans, the traffic wardens would often leave their duty post to attend to hunger, thirst, nature's call; to take shelter from inclement weather; or at the end of their shift. Over time, traffic lights, also known as traffic signals, traffic lamps, traffic semaphore, signal lights, stop lights, and traffic control signals (in technical parlance) were introduced and positioned at road intersections, pedestrian crossings, and other locations to control traffic flow. Traffic lights alternate the right of way accorded to road users by displaying lights of a standard colour (red, yellow/amber and green) following a universal colour code. 
The use of traffic lights has offered tremendous benefits compared to the use of traffic wardens, in that it reduces manpower and has significantly reduced chaos at road intersections. As of date, there are two major traffic management approaches: fixed time and adaptive. In the fixed time scheme, a team of experts decide the traffic control sequence that will apply. They can choose to operate the traffic light using a fixed-time control all through the day or slightly vary the traffic control sequence based on their observations of the geographical area per time of day. Despite their best efforts, it is not uncommon to find traffic congestion on the more operational lane while an apparently empty lane is being allotted its pre-programmed right of way. This is an indication that traditional methods of modelling and control cannot work very well for traffic control.

In the adaptive scheme, sensors are used to measure the real-time traffic density. This information is subsequently used to alter the traffic control sequence to ensure the more operational lanes are allotted slightly longer time than the less operational lanes. Two types of sensors are commonly used: above-ground sensors (video image processing, microwave radar, laser radar, passive infrared, ultrasonic, and passive acoustic array), and underground sensors (inductive loops, micro-loop probes, and pneumatic road tubes). Some of these sensors are bulky, power-hungry, and expensive to install, maintain and repair. In addition, the accuracy of some of them varies with the climate.

Typically, a set of sensors is used to count the number of vehicles passing the traffic lights at a road intersection while another set of sensors is used to count the number of vehicles approaching the road intersection at distance $D$ from the traffic lights. The traffic density can be computed from the difference in the two set of readings. Similarly, the difference in the timestamp of each set of readings can be used to compute the traffic flow and waiting time. Fundamentally, a Wireless Sensor Network (WSN) can be set up to efficiently handle communication between the various sensor modules. A WSN consists of sensors and gateway nodes. The duty of sensor nodes in a traffic management system (TMS) is to monitor traffic in an allocated area by measuring physical traffic parameters like flow, density, waiting time, throughput, as well as emergency (Chao \& Chen, 2014). The gateway node collects traffic information from all the nodes and directs same to the base station.

A TMS can be designed to be interrupted using button actuators to give priority to special traffic, usually emergency vehicles such as: fire apparatus, ambulances, and police squad cars. At such instances, the normal traffic light sequence is temporarily overridden with the special sequence. Usually, there is an additional signal light placed near the traditional red-amber-green lights to indicate that the special sequence has been activated and to warn other motorists of approaching emergency vehicle(s). A TMS can also be designed to automatically detect special vehicles, especially mass transit vehicles and make small adjustments to the traffic control sequence. Modern Artificial Intelligence (Al) algorithms can be utilized to either decrease the likelihood that a mass transit vehicle will arrive during a Red interval or decrease the duration of the Red interval as the mass transit vehicle approaches.

\subsection{Statement of Problem}

An analysis of the current TMS in Uyo, an urban city in Nigeria showed a heavy dependence on the use of traffic wardens. Where traffic control lights operate, they are based on fixed time allocation. The absence of sensor-based solutions often results in traffic congestion on the more operational lane while an apparently empty lane goes through its pre-programmed cycle. 
More importantly, many of the sensor-based TMS proposed in extant literature focus primarily on improving the traffic flow on all lanes; few consider the need to reduce or eliminate the waiting time of emergency vehicles to safeguard against loss of lives and properties.

\subsection{Objective}

This study explores the design of a model which uses passive Infrared (IR) sensors to measure traffic parameters, such waiting time and traffic density; a button actuator to signify the presence of an emergency vehicle; a Programmable Integrated Circuit (PIC) for intelligent traffic light control; and a blue signal light to indicate when the special traffic control sequence has been activated.

\section{RELATED WORK}

Intelligent TMS can be grouped depending on the approach used: centralized or de-centralized. In the centralized approach, sensors (e.g., induction loops, video cameras, and radar) are placed at specific locations along a road to acquire traffic data. In the de-centralized approach, traffic data is crowd-sourced from passing vehicles in an ad hoc manner through the inter-networking of vehicles, often termed a vehicular ad hoc network (VANET) made possible through the use of short-range radios, e.g., Wi-Fi or dedicated short-range communication (Leontiadis, Marfia, Mack, Pau, Mascolo \& Gerla, 2011). The centralized approach is adopted in this study.

Five key requirements are considered important in the design of a TMS: multi-modal sensing, real-time communication, interoperability, reliability, and security (Kafi, Challal, Djenouri, Bouabballah, Khelladi \& Badache, 2012). Firstly, multi-modal sensing is required to ensure the TMS utilizes various environmental parameters, such as: gas emissions, number of stops, and noise levels due to traffic in its intelligent decision making. Secondly, real-time communication is required to ensure the TMS is fed with real-time measurements of traffic parameters, such as waiting time and traffic density. Thirdly, interoperability is necessary to ensure that where diverse TMSs were implemented at different road intersections in a geographical area, they can be integrated to provide a global solution across that geographic area regardless of hardware heterogeneity. Fourthly, a reliable end-to-end communication ensures data reliability regardless of the lossy communication channel, low coverage, and limited capacity of the WSN. Lastly, a highly secure communication safeguards against attacks by hackers and terrorists, prevents denial of service through jamming, and guarantees service continuity in the event of a failure.

A comparative study of four intelligent TMS algorithms: Equal Interval, Optimum Equal, Webster and Dynamic Webster was conducted by Al-Kandari, Al-Shaikhli and Najaa (2013). The Equal Interval algorithm is the simplest and most straight forward of the four methods as it gives each traffic light an equal Green Interval value. The Optimum Equal algorithm is similar to the Equal Interval but differs in how it calculates the Actual Cycle Interval by multiplying the sum of the queues by the average cross ratio and subtracting the wait time. What this means is instead of giving static Cycle Interval that does not scale with traffic load, the system decides to give Dynamic Cycle Interval that scales with the Queues as well as the average of the Cross Ratio. The Webster method calculates the actual cycle interval just like Equal Interval. However, it calculates the Green Interval depending on the current queue relative to the total queue number of all traffic lights. This is somehow similar to Optimum Equal but instead of looking at the intersection as a whole "Total Queue", the method focuses on each Queue and the percentage of cars that exist in it and give it a suitable value out of the $100 \%$ of the Green Interval Time. 
The Dynamic Webster method is identical to the Webster method except that it does not wait until cycle completion before calculating the Total Queue (as the Webster does). Rather, it calculates the Total Queue continuously and resets the Green Interval for each traffic light accordingly. A random function was used to add cars to each lane at random intervals so as to provide a realistic simulation. Findings show that the Dynamic Webster out-performed the other algorithms. Relative to the flow rate achieved by Dynamic Webster, Webster recorded 99\%, followed by Optimum Equal 93\%, and lastly by Equal Interval 90\%.

The system proposed by Collota, Giuffre, Pau and Scata (2014) consist of a hierarchical network characterized by two layers: one layer is represented by a wireless sensor network based on the IEEE 802.15.4 protocol while the other layer consists of a wired backbone. The IEEE 802.15.4 protocol, in beacon-enabled mode, makes possible a priori scheduled communication using Guaranteed Time Slot (GTS). The use of GTS allows to have a communication as deterministic as possible; however, not more than seven devices can be accommodated at the same time. The dynamic management algorithm is divided into two steps. In the first step the phase sequence is determined. Based on the queue length for each flow (input variable), the algorithm assigns a priority level to each phase equal to the maximum queue length of that phase. Thereafter, the algorithm determines the phase sequence by sorting them in descending order priority.

In the second step, the green signal time is calculated. The algorithm, for each flow, processes the number of vehicles passed during the previous traffic light cycle and uses this value to determine the current traffic volume. This value is used to re-calculate the green time duration of the next cycle based on traffic detected. This approach was evaluated in a simulator developed in $\mathrm{C}$ language. Results show that the algorithm is capable at optimizing traffic flow at road intersections which experience irregularly traffic flows at various times of the day. One of the recommendations made by Collota et al. (2014) is for the system to be improved to enable priority passage of emergency vehicles and preferential priority passage of mass transit vehicles.

When a plethora of sensors are utilized to measure traffic parameters, the malfunctioning of some sensors complicates the decision architecture and reduces its overall accuracy. So the fewer the number of participating sensors, the more reliable the system is likely to be. Alternatively, rather than utilizing a plethora of low cost sensors which are likely to be less reliable, the TMS can be implemented using a mixture of high cost sensors and reliable expert systems. To keep cost within a reasonable amount, Pescaru and Curiac (2014) suggested that high cost traffic sensors should organized in an ensemble decision network and placed only on the exit of the last intersection for all the principal roads entering a city zone.

This is because, typically, only a small number of roads entering a city zone coagulate the majority of traffic. The principal road (major road) is a high-capacity street that attracts traffic from the surrounding areas. The other roads in the zone, with less traffic, are called side roads. A principal crossroad is an intersection between a minimum of two principal roads. The rest of the crossroads are regarded as secondary intersections. In our daily life, it is typical to find a person obtaining two, three or even more qualified opinions whenever a complex decision is to be made. An ensemble of classifiers work in a similar manner. 
By utilizing an ensemble decision network, hardware or analytic redundancy can be implemented to cater for cases where some traffic sensors are working incorrectly - either by installing more than one sensor on each principal road, or by computing an estimated value for that sensor using measurements obtained from other sensors. WSNs are very trendy due to their faster transfer of information, easy installation, less maintenance, compactness and for being less expensive compared to other network options. In a survey of urban traffic management schemes designed using WSN for priority-based signalling, reducing congestion, as well as reducing the average waiting time (AWT) of vehicles, Nellore and Hancke (2016) noted that 10 different kinds of traffic sensing technologies were utilized in extant literature to monitor real-time traffic. These are: inductive loop, Radio-Frequency Identification (RFID), microwave radar, acoustic, magnetometer, magnetic induction coil, infrared, aerial/satellite imaging, ultrasonic, and video image processor. Nellore and Hancke (2016) also noted that eight different kinds of wireless communication technologies were utilized. These are: Wi-MAX, ZigBee, Bluetooth, UWB, Wi-Fi, GSM, GPRS, and RFID.

When RFID technology is used to grant priority to special vehicles in a TMS, the system can be designed such that whenever an emergency vehicle approaches a signalized intersection, the RFID reader reads the RFID tag mounted on the emergency vehicle and sends a signal to the traffic controller. In the system proposed by Bhavana and Nagamani (2018), data from the RFID reader were communicated using ESP8266 Wi-Fi module to ThingSpeak, an Internet of Things (loT) cloud service, from which signals were transmitted to Arduino ATmega2560 microcontroller which served as the traffic light controller.

If medical aid can be administered to a patient within the first hour of an emergency, there is a greater likelihood of saving the patient's life. Sarpal, Asthana and Hota (2020) noted that about $30 \%$ of deaths arising from road accidents could have been prevented if an ambulance had reached the victim in good time. Similarly, more than $50 \%$ of heart attack cases would not have resulted in death if an ambulance had reached the patient in good time.

Typically, concrete pavements or a bed of grass (with or without interlacing trees surrounded by concrete blocks) is used for contra-traffic separation in a two lane dual carriage road (i.e., four lanes in total) while asphalt markings is used to demarcate lanes along the same traffic direction. This approach is fixed and difficult, if not impossible, to alter as traffic demand varies. Prasanna, Menaka, Ram and Rithul (2020) suggested that adjustable pole blockades, controlled using loT, could be used to allocate three lanes to the more operational lane (and especially when emergency vehicles are present) while the less operational lane takes the remaining one lane. In their proof-of-concept, real-time traffic parameters were obtained using video cameras processed using computer vision techniques on MATLAB software. The system was programmed using python language with the help of OpenCV.

When a TMS is implemented using image processing techniques, a digital camera captures images at a road intersection when it is completely clear (i.e., no traffic). These blank street images are converted from RGB to Gray and subjected to gamma-correction to that they can optimally serve as source (reference) images. To determine the real-time traffic density, the current images of the road intersection are also obtained, converted from RGB to Gray, subjected to gamma-correction and compared with the reference images. In image processing, the edges of an image are usually found at the intersection of two separate regions in an image. Edge detection helps to determine the areas in an image where the Gray level suddenly changes, signalling the end of one area and the start of another. 
In the system proposed by Al-Abaidy (2021), edge detection was performed using Prewitt edge detection algorithm. Further, IR sensors were incorporated to ensure that vehicles have thoroughly exited the road intersection before the next traffic light sequence kicks in. Given the near ubiquity of smart mobile devices (tablets, smart phones, and smart watches), Bhosale, Kamble, Mane and Deshmukh (2021) proposed a TMS that enables an ambulance driver to send a signal to traffic light controller through the Internet using an Android based mobile app rather than a button actuator. The traffic light controller was designed using Arduino UNO board interfaced with ESP8266 Wi-Fi module. The system has a central server which serves to connect the traffic light controller and the ambulance. The API and central server were created in the Google cloud service platform.

\section{CONCEPT DESIGN}

\section{A. Research Design}

Research methodology is a way to systematically solve a research problem. It outlines the various steps adopted by a researcher in studying a research problem alongside the underlying logic necessitating the choice of those steps so that research results can be readily evaluated either by the researcher or others. The Dynamic Systems Development Method (DSDM) guided the development of this TMS-ETD model. DSDM is a framework for software development in an agile way with clearly defined principles, roles, phases, and sub-phases. It bears many similarities with other agile methods such as Scrum and Extreme Programming.

Four variables were considered important: (i) information on the active phase; (ii) information on the last green light duration; (iii) information on the time step for the determination of the next phase; and (iv) information on the time when the duration of the phase has to be set, i.e., when the green phase has to be changed. Since the algorithm does not calculate the next cycle but only the duration of the next phase, these four variables have to be kept in memory and used when the traffic light program is at the beginning of the determined phase to be able to set the calculated duration.

\section{B. System Design}

Fig. 1 depicts the architecture of the TMS-ETD model in the simulation environment of Proteus 8 professional version 8.6. The model consists of four units: Microcontroller Unit (MCU), Traffic Density Unit (TDU), Emergency Signal Unit (ESU) and Traffic Light Indicator (TLI) unit. In the simulation, switches are used to mimic roads and vehicles. There is a switch for each of the four lanes. Whenever a switch is closed, it implies that the lane so represented has the highest traffic density. Switches are also used to represent the emergency button actuators. Whenever a button actuator is pressed, it implies that an emergency vehicle is requesting right of way for its lane. Four colours (Red, Yellow, Green and Blue) are used as TLIs. The MCU processes input signals from all the switches and buttons and makes intelligent decisions that are displayed as output on the TLI unit. 


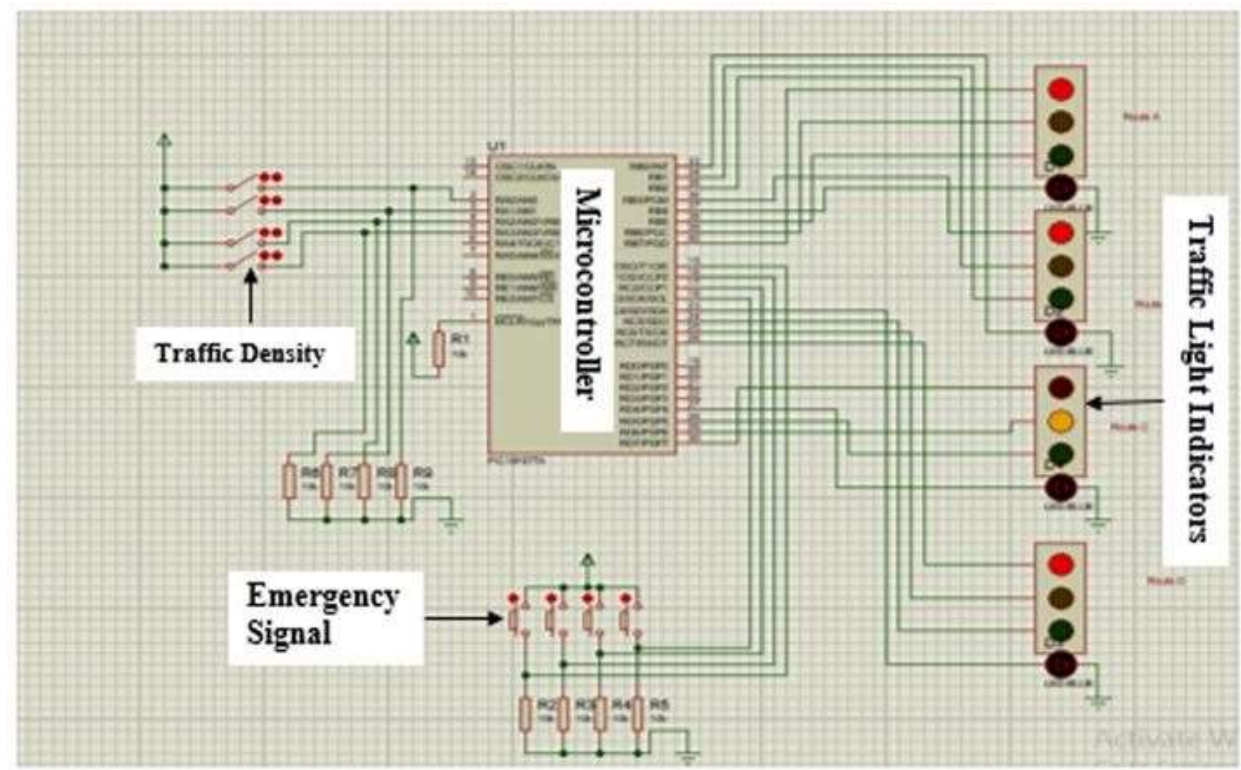

Fig. 1: Architecture of TMS-ETD

\section{Mathematical Model}

The notations depicting directions, lanes and cases are given as:

$$
\begin{aligned}
& D=\{\text { North, South, East, West }\} \\
& L=\{\text { Forward, Right }\} \\
& C=\{1,2,3,4 \ldots 12\}
\end{aligned}
$$

where $D, L$ and $C$ denote the set of directions, lanes and cases respectively.

Expectant Phase $(E \varphi$ of a vehicle is defined as the phase in which a vehicle passes a road intersection.

$$
\begin{aligned}
& \text { Case 01: } E \varphi(N, F)=E \varphi(N, R)=\varphi a \\
& \text { Case 02: } E \varphi(S, F)=E \varphi(S, R)=\varphi b \\
& \text { Case 03: } E \varphi(E, F)=E \varphi(E, R)=\varphi c \\
& \text { Case 04: } E \varphi(W, F)=E \varphi(W, R)=\varphi d \\
& \text { Case 05: } E \varphi(N, F)=E \varphi(S, F)=\varphi e \\
& \text { Case 06: } E \varphi(N, R)=E \varphi(S, R)=\varphi f \\
& \text { Case 07: } E \varphi(E, F)=E \varphi(W, F)=\varphi g \\
& \text { Case 08: } E \varphi(E, R)=E \varphi(W, R)=\varphi h \\
& \text { Case 09: } E \varphi(N, F)=E \varphi(W, R)=\varphi i \\
& \text { Case 10: } E \varphi(S, F)=E \varphi(E, R)=\varphi j \\
& \text { Case 11: } E \varphi(N, R)=E \varphi(E, F)=\varphi k \\
& \text { Case 12: } E \varphi(S, R)=E \varphi(W, F)=\varphi l
\end{aligned}
$$

Lane Waiting Queue, denoted as $Q(d, I)$, is defined as the number of vehicles waiting on the path $P$.

$$
Q\{d, l\}, d \in D \text { and } I \in L
$$


Phase Waiting Queue $(\mathrm{Qx})$ is the length of queue for phase $\mathrm{x}$.

$Q x=\operatorname{MAX}(T Q(d, I))$

where $(d, l) \in\{E \varphi(d, l)=\varphi x\} ; d \in D ; \mid \in L ;$ and $x \in\{a, b, c, \ldots, l\}$.

Queue passing time, represented as TQ $(d, I)$, is the time taken by all waiting vehicles to pass the intersection.

$T Q(d, l)=T 1+(T *(Q(d, l)-1))$

where $\mathrm{T} 1$ is the time taken by first vehicle to cross the intersection; and $\mathrm{T}$ is the time taken by a vehicle to move to the place of front vehicle.

Phase Queue passing time (TQx) is calculated as:

$\mathrm{TQx}=\operatorname{MAX}(\mathrm{TQ}(\mathrm{d}, \mathrm{I}))$

where $d, I \in\{E \varphi(d, I)=\varphi x\} ; d \in D ; I \in L ; x \in\{a, b, c, \ldots, \mid\}$.

Emergency condition, denoted as EC (d, I), determines the presence of any kind of emergency vehicle.

Waiting Time, denoted as WT $(\mathrm{d}, \mathrm{l})$, gives the waiting time for the vehicle in front.

Threshold waiting time (Threshold) sets the maximum waiting time.

Phase time (TPx) defines the duration of Green light for Phase x.

$$
\text { TPx }=\text { MIN (TQx, TPmax })
$$

Maximum Phase Time (TPmax) gives the maximum time for which Green light is allotted.

In this study, emergency vehicles have been categorized and assigned priorities as follows: fire truck - 0 (highest); ambulance - 1; police patrol - 2 (lowest). This categorization can be modified in line with the prevailing policy in any country. Considering all probable scenarios, emergency vehicles can be allocated priority passage based on the following four cases:

CASE 1: Only one emergency vehicle is present This is the simplest scenario which simply requires a green light being assigned to the lane where the emergency vehicle is present.

CASE 2: Two emergency vehicles each having different priority In this case, the vehicle with the higher priority is first given attention.

CASE 3: Two emergency vehicles each having same priority In this case, the vehicle with the shortest distance from the intersection is first given attention.

CASE 4: Four emergency vehicles (all different priorities) on each lane (DEADLOCK) This is the most complex scenario which can be tackled using the Highest-priority First Scheduling (HFS) method. 


\section{Algorithm}

Fig. 2 below describes the algorithm of the TMS-ETD system. The major steps are as follows:

Step 1: Start

Step 2: Normal mode

This mode measures the traffic density and activates traffic lights accordingly.

Step 3: Check the incoming message

If message is from an emergency vehicle, then change to emergency operation mode. If accident message from traffic control officer, then change to accident operation mode. If force ending message, then stop system. If not, loop to normal mode.

Step 4: End

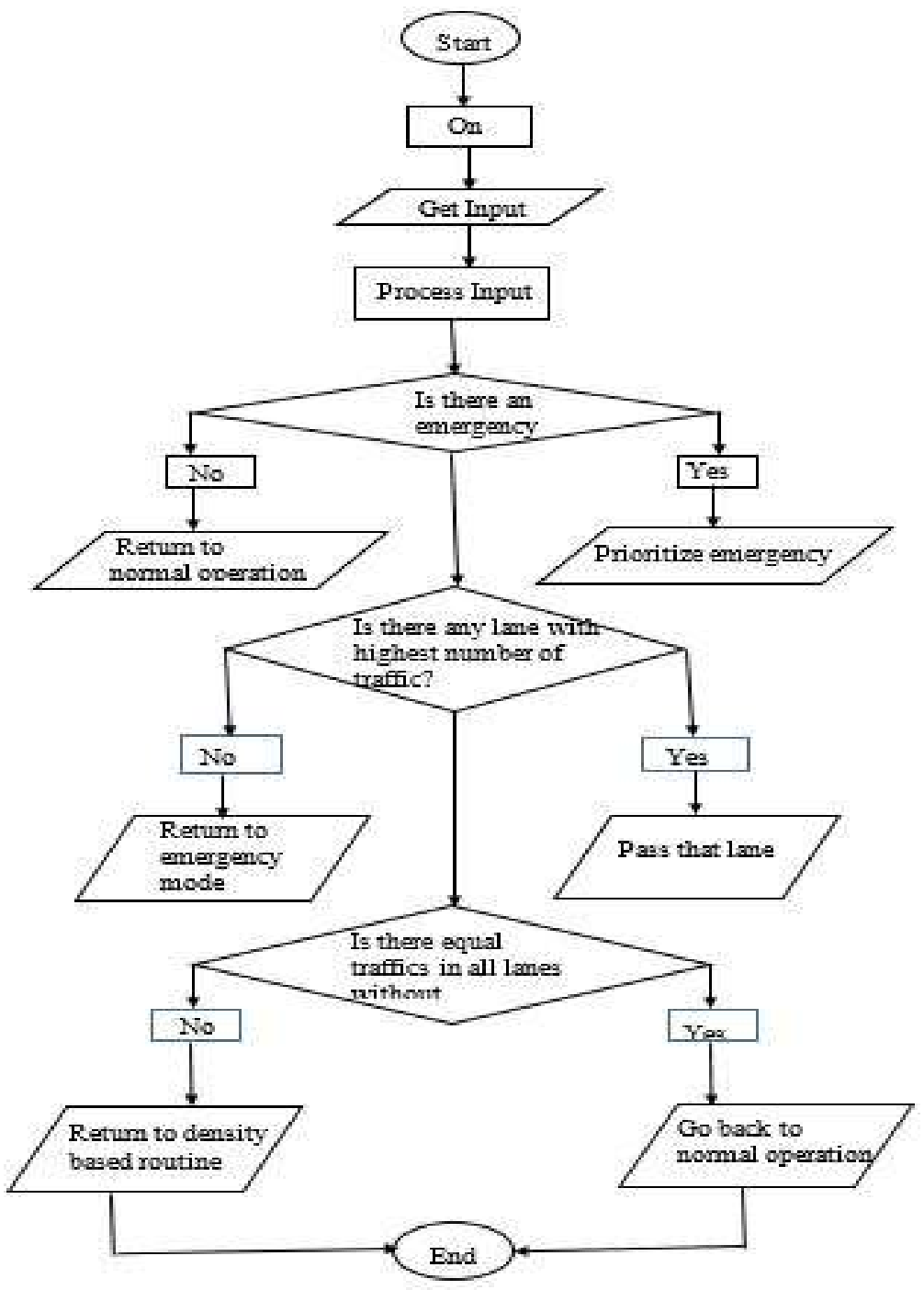

Fig. 2: Flowchart of TMS-ETD model 


\section{E. Testbed}

As proof-of-concept, the TMS-ETD model was implemented on a testbed (Fig. 3). The following components were used: PIC16F770A microcontroller, programmed using MikroC PRO for PIC version 6.0.0; HC-SR505 passive Infrared (IR) sensor for acquiring real-time traffic data; HC-05 Bluetooth module for wireless communication; 6F22 9V battery for power supply; and red, green, yellow and blue Light Emitting Diodes (LEDs) as traffic light indicators.

The infrared sensor illuminates the low powered infrared energy in the recognition regions and captures the echoed energy from the vehicles. The echoed energy is focused onto an infraredsensitive material, which transforms the echoed and illuminated energy into electrical signals. These signals are processed and analysed to obtain the presence of a vehicle. Since infrared sensors are sensitive to bad weather and require periodic lens cleaning, a multi spectrum camera can be used to obtain more accurate traffic parameters.

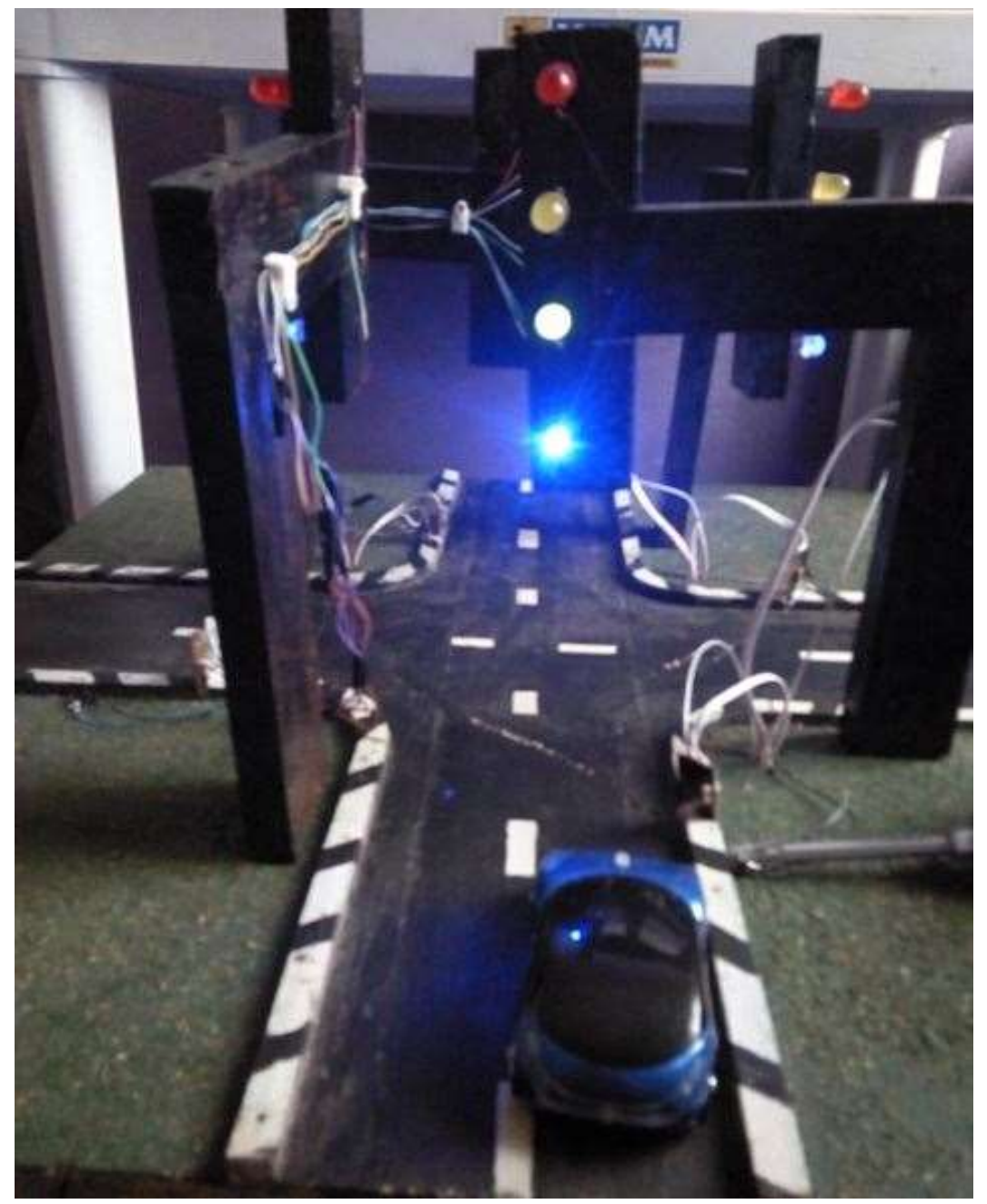

Fig. 3: Test Bed of TMS-ETD

The heart of the system is the PIC16F877A microcontroller which implements the basic operations. Additional ports and multiplexers were used to enable communication with the 
external devices. Additional RAM and ROM were used to store system program and application program. Other components used in the proof-of-concept include: input switching matrix, serial communication interface, clock circuit, and LED interfacing circuit.

The signals from the sensors are applied to the input switching circuit. The input signals from the sensors is in the form of digital signals which corresponds to presence or absence of a vehicle. These digital signals from each lane are supplied to the input port of the microcontroller, where the microcontroller determines the numbers of vehicle at each lane. This helps the microcontroller to ascribe various timing sequences to each lane accordingly. The input signals are applied to two relay drivers. The relay drivers are level shifters and current amplifiers. Lastly, the output of the relay driver is applied to the TLIs (Red, Green, Yellow and Blue LED).

\section{RESULTS}

This TMS-ETD model is capable of adapting the traffic signal time at road intersections by utilizing real-time traffic density data obtained using IR sensors placed at the road side near the traffic control lights. The model was simulated for an isolated four-way road intersection with varying traffic density on each of the four lanes. Most importantly, the underlying algorithm adapts the cycle intervals to ensure minimum waiting time for the lane with emergency vehicles before considering the lane with the longest waiting time and lastly the lane with the highest traffic density.

\section{CONCLUDING REMARKS}

The passage of emergency vehicles is often ignored in the design of most traffic management systems leading to avoidable loss of lives and properties. This study explored the design of a traffic management system capable of reducing/eliminating the waiting time of emergency vehicles at busy road intersections, as well as prioritizing the more operational lanes over the lesser operational lanes. A testbed designed using PIC 16F770A microcontroller programmed using MikroC PRO version 6.0.0 for processing raw input data and HC-SR505 IR sensor for measuring traffic density was constructed to demonstrate the practicability of this model. The model was simulated using Proteus 8 where results showed a higher potential for curbing traffic congestion as well as safeguarding lives and properties.

\section{RECOMMENDATION AND FUTURE WORK}

\section{A. Recommendation}

The TMS-ETD model can be implemented at any busy road intersection in towns and major cities of the world to reduce congestions and prioritize the passage of emergency vehicles. To ensure smooth operation of the system, the choice of sensors should take into consideration the prevailing environmental conditions at the deployed location. Also, there should be provision of a back-up power source at locations where intermittent power supply is prevalent. Lastly, the actuator buttons can be integrated with the command and control system of emergency services to prevent first responders utilizing the button when they are not actively responding to an emergency situation 


\section{B. Future Work}

This TMS-ETD model is most suitable as a local strategy to cater for isolated road intersections. However, nowadays, city planners are more interested in global strategies able to ease traffic flow across neighbouring road intersections in a geographical area. This can be achieved by devising a more robust TMS-ETD model.

In addition, future enhancement to the TMS-ETD model can be made to support easy integration with other smart city initiatives to enable citizens derive additional benefits, such as: route planning to minimize fuel consumption, cost and time; pollution reduction to minimize impact on environment; road condition updates to highlight areas in need of urgent maintenance and repair; public transport management; and road tax enforcement.

By extending the TMS-ETD framework to incorporate the use of on-vehicle sensors, a broad range of smart city applications can be supported. For instance, travel tolls can be enforced by capturing the identity of every vehicle passing at an intersection. Similarly, speed regulations can be enforced by capturing the time stamps of a vehicle at each node in a network of intersections. Any vehicle that arrives at a neighbouring node much shorter than the average time can be tracked down and the driver fined for over-speeding. In addition, vehicles that cross an intersection at any time other than during the Green Interval can be tracked down and the driver fined for reckless driving.

\section{REFERENCES}

1. Viswanathan, V. \& Santhanam, V. (2013). Intelligent Traffic Signal Control Using wireless Sensor Network. Proceeding of 2nd International Conference on Advances in Electrical and Electronics Engineering. pg. 165-169.

2. Ovadje, F. (2021, Feb 22). Economic Costs of Traffic Congestion in Lagos. Lagos: Danne Institute for Research. Accessed on 27 August 2021, 11:45am from: https://danneinstitute.org/publications/always-go-with-a-smile-4/

3. Chao, K. H., \& Chen, P. Y. (2014). An intelligent traffic flow control system based on radio frequency identification and wireless sensor networks. International Journal of Distributed Sensor Networks, 10(5), 694545.

4. Kafi, M. A., Challal, Y., Djenouri, D., Bouabballah, A., Khelladi, L., Badache, N. (2012). A study of wireless sensor network architectures and projects for traffic light monitoring. Proceedings of the 3rd International Conference on Ambient Systems, Networks and Technologies (ANT), Procedia Computer Science, 10, pp. 543-552. DOI 10.1016/j.procs.2012.06.069

5. Leontiadis, I., Marfia, G., Mack, D., Pau, G., Mascolo, C., \& Gerla, M. (2011). On the Effectiveness of an Opportunistic Traffic Management System for Vehicular Networks. IEEE Transactions on Intelligent Transportation Systems, 12 (4), pp. 1537-1548. DOI 10.1109/TITS.2011.2161469

6. Al-Kandari, A., Al-Shaikhli, I., \& Najaa, A. (2013). Comparative study between traffic control methods using simulation software. International Journal of Machine Learning and Computing, 3(5), pp. 424-429. DOI - 10.7763/IJMLC.2013.V3.353

7. Collota, M., Giuffre, T., Pau, G., \& Scata, G. (2014). Smart Traffic Light Junction Management Using Wireless Sensor Networks. WSEAS Transactions on Communications, 13, pp. 648-658 
8. Pescaru, D., \& Curiac, D.I. (2014). Ensemble based traffic light control for city zones using a reduced number of sensors. Transportation Research Part C, pp. 261-273. DOI - http://dx.doi.org/10.1016/j.trc.2014.06.006

9. Nellore, K., \& Hancke, G. P. (2016). A survey on urban traffic management system using wireless sensor networks. Sensors, 16(2), 157, pp. 1-25. DOI -10.3390/s16020157

10. Bhavana, G. R. \& Nagamani, K. (2018). Implementation of Intelligent Traffic Management System using loT. International Journal of Electrical Engineering and Technology (IJEET), 11(5), pp. 22-30. DOI - 10.34218/IJEET.11.5.2020.003

11. Prasanna, K. R., Menaka, R., Ram, P. P., Rithul, M. (2020). Implementation of high performance traffic management system using novel blockade mechanism. IOP Conference Series: Materials Science and Engineering, 994, 012028, pp. 1-14. DOI 10.1088/1757-899X/994/1/012028

12. Sarpal, D., Asthana, Y., and Hota, M. K. (2020). Review on Smart Traffic Management System for Ambulance.International Journal of Electrical Engineering and Technology (IJEET), 11(6), pp. 1-9. DOI - 10.34218/IJEET.11.6.2020.001

13. Al-Abaidy, S.A.F. (2021). Intelligent traffic management systems using image processing techniques. Turkish Journal of Computer and Mathematics Education, 12(7), pp. 258262.

14. Bhosale, D. A., Kamble, R., Mane, M., \& Deshmukh, A. (2021). Smart traffic management system. International Journal of Research Publication and Reviews, 2(2), pp. 226-228. 